\title{
Experience with parallel optical link for the CDF silicon detector
}

\author{
S. Hou for the CDF optical readout group \\ Institute of Physics, Academia Sinica, Taipei 115, Taiwan
}

\begin{abstract}
The Dense Optical Interface Module (DOIM) is a byte-wide optical link developed for the Run II upgrade of the CDF silicon tracking system [1]. The module consists of a transmitter with a laser-diode array for conversion of digitized detector signals to light outputs, a $22 \mathrm{~m}$ optical fiber ribbon cable for light transmission, and a receiver converting the light pulses back to electrical signals. We report on the design feature, characteristics, and radiation tolerance.
\end{abstract}

The DOIM optical links [2] are used to replace conventional copper wires for high bandwidth data transmission with low-mass optical fibers in a harsh radiation environment for the CDF silicon tracker readout. The data transfer rate is $53 \mathrm{Mbyte} / \mathrm{sec}$ per module and the radiation tolerance is required for $200 \mathrm{krad}$. The transmitter module consists of an edge-emitting laser-diode array (LDA) and a custom driver chip. The laser wavelength is $1550 \mathrm{~nm}$. The receiver module has a matching PIN-diode array (PDA) and a chip for converting optical inputs to differential ECL signals. Each transmitter and receiver pair has nine identical channels for transmission of 8-bit data and a clock signal.

The laser diode and PIN diode arrays are 12-channel InGaAsP/InP devices of $250 \mu \mathrm{m}$ pitch fabricated by the Telecommunication Laboratories of Chunghwa Telecom [3]. The driver chips were fabricated by the AMS $0.8 \mu \mathrm{m}$ BiCMOS process [4] using bipolar transistors for high speed capability and good radiation tolerance. The module assembly contains a short optical fiber pigtail terminated with a standard MT type connector for transmitting or receiving optical signals. Pictures of complete modules are shown in Fig. 1. The type of fiber used for pigtails and cables is a graded-index multimode fiber of Plasma Optical Fibre [5] with germanium doping. The core diameter is $62.5 \mu \mathrm{m}$ and the cladding diameter is $125 \mu \mathrm{m}$. The fibers were coated and bundled into 12-channel ribbons with a pitch of $250 \mu \mathrm{m}$. 

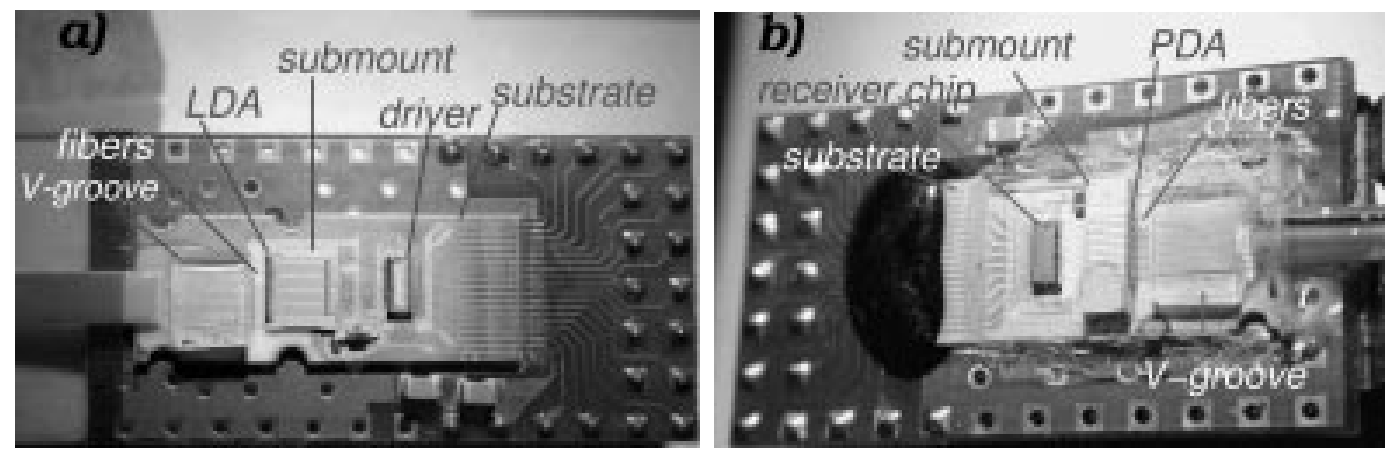

Fig. 1. Pictures of (a) a transmitter and (b) a receiver module on carrier boards. The covers were removed.

The signal inputs to the transmitter driver chip are in LVDS format with a common level around half the chip bias voltage $\left(V_{c c}\right)$. The laser diodes are connected to switching circuits terminated with a common voltage level $V_{L D}$. The nominal switch-on current for a laser channel is $20 \mathrm{~mA}$. The laser current is proportional to the voltage drop and is adjusted by tuning $V_{L D}$ at the level of $2 \mathrm{~mA} / 0.1 \mathrm{~V}$. The receiver module has a low input threshold of $50 \mu \mathrm{W}$ and a non-saturating dynamic range up to $800 \mu \mathrm{W}$. The laser light pulses received by the PIN diodes are converted to current signals to the receiver chip. The outputs are in standard differential ECL format.

The electrical characteristics of the transmitter module were investigated using a bias scheme, shown in Fig. 2.a, compatible to the circuit design for CDF. The signal inputs were given by a function generator, and the enable line was controlled by a TTL signal for switching off all laser diodes with a level high. The pigtail was connected to a MT to 12-ST fiber fanout. The light output of a fiber channel was measured with a Optical-Electrical probe. A typical oscilloscope image for the inputs and light output is shown in Fig. 2.b. The

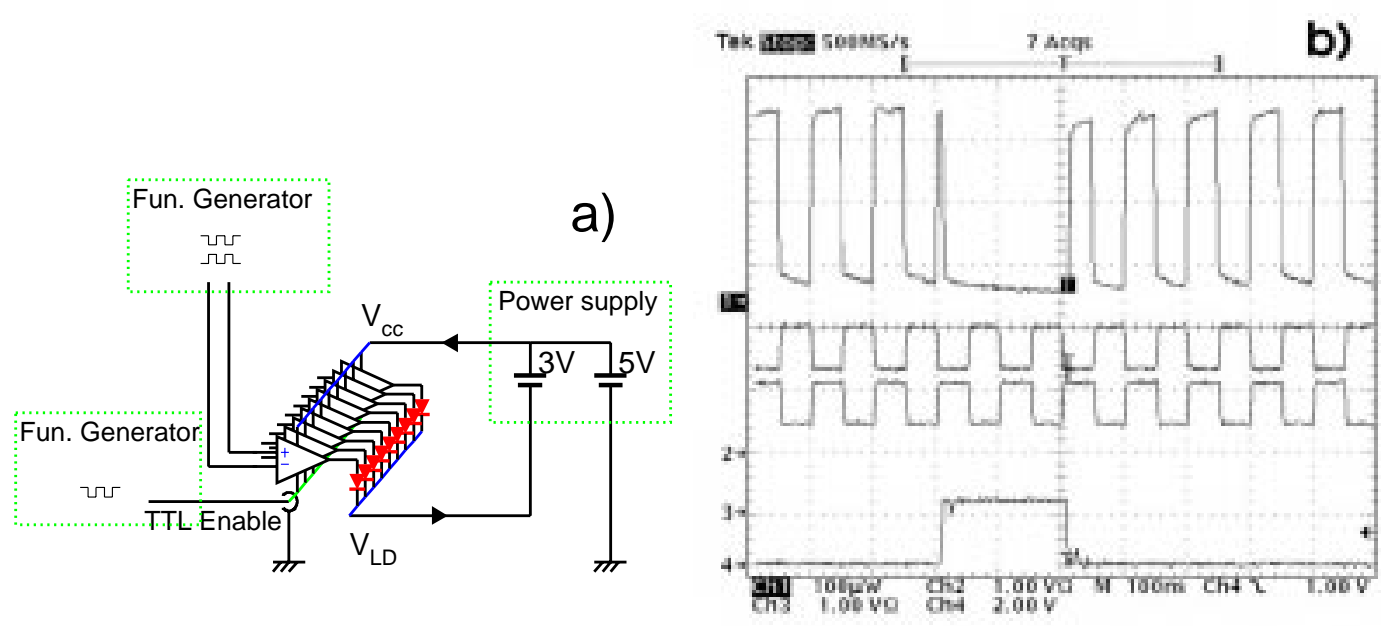

Fig. 2. (a) Schematics for the laser transmitter test setup, and (b) a typical oscilloscope image obtained. The inputs to the transmitter are the TTL enable (bottom line) and the LVDS signals (middle two lines). Upper line is the light output of a laser diode channel measured with a Tektronix P6703B Optical-Electrical probe. 
power dissipation is about $2 \mathrm{~W}$ and the current flow from $V_{c c}(+5 \mathrm{~V})$ is stable at about $160 \mathrm{~mA}$. The I-V curves measured for a typical laser diode are shown in Fig. 3.a for three temperature settings measured at the substrate edge. The temperature is typically maintained at a few tenth of a degree Celsius in precision on a water cooling system. The light outputs for a laser channel are plotted in Fig. 3.b. The temperature dependence of laser light level is approximately linear, the correlation is shown in Fig. 3.c.

The DOIM components were selected by a series of burn-in and test procedures prior to the module assembly. The complete modules were then tested using the Bit Error Rate Tester (BERT) [6] developed at Fermilab. The tests were performed with random data pattern downloaded on the test boards, then transmitted and checked on-the-fly at a chosen frequency. The bit error rate is required for less than $10^{-12}$. The BER tests were normally running at $63 \mathrm{MHz}$ for a time duration a few times longer than required. The tests are effective in purging weak modules having problems in assembly. Modules with defects usually fail instantly. Qualified modules can last in the tests for weeks without an error.

The transmitter modules in use at CDF are subject to radiation damage for a total dose of $200 \mathrm{krad}[1,7]$. The degradation in light power is considered mostly due to displacements of ions in laser diodes. Irradiation studies were performed with $30 \mathrm{MeV}$ protons at the Institute of Nuclear Energy Research (INER) in Taiwan at a fluence rate of $1.4 \times 10^{10}$ proton $/ \mathrm{cm}^{2} \mathrm{~s}$. The beam spot was distributed in a $2 \mathrm{~cm}$ diameter area. The beam current was stable and was monitored by a Faraday cup device to an accuracy of better than $2 \%$. The transmitters were submitted one at a time with the pigtail connected to
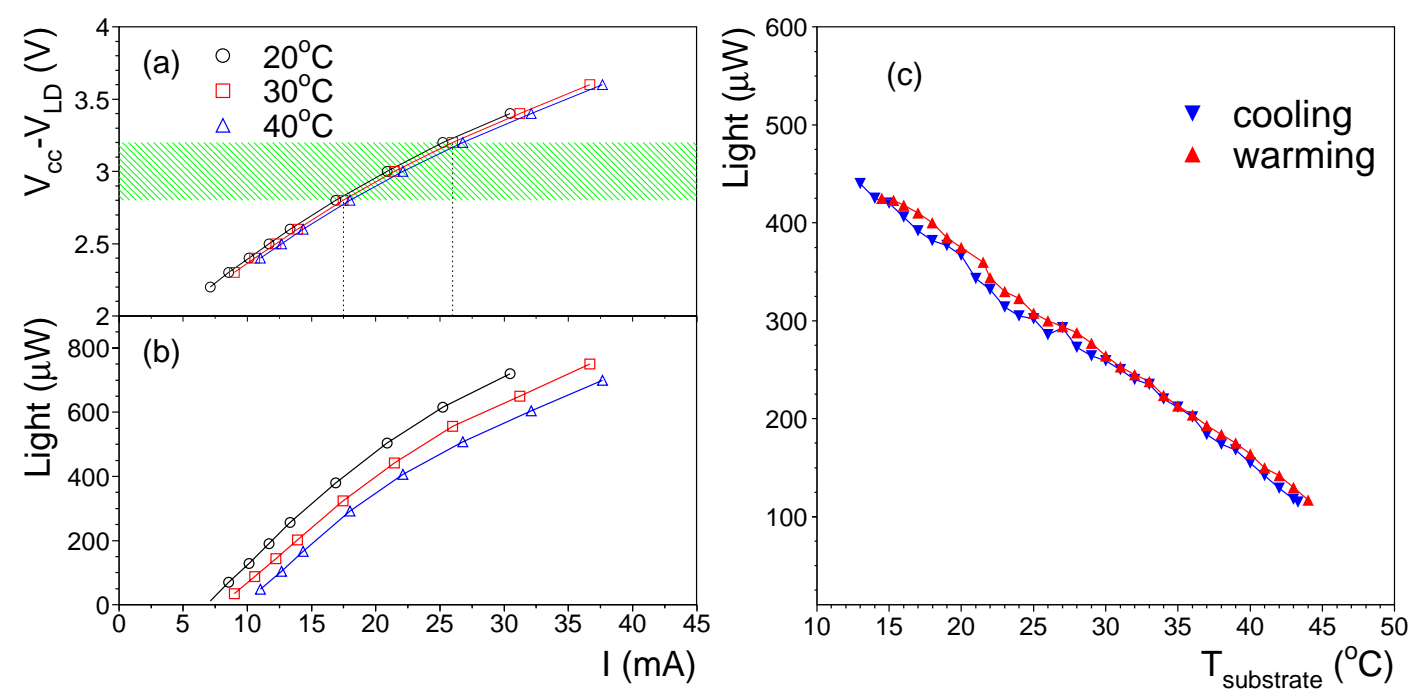

Fig. 3. The laser-diode characteristics are shown for (a) voltage drop $V_{c c}-V_{L D}$, and (b) light output versus average current consumption for a laser channel. The hatched area is the desired operation region. The temperature indicated was measure at the substrate edge. The temperature dependence for light output is shown in (c) for a laser diode measured in steady cooling and warming cycles. 
a fiber ribbon cable out of the test area and was measured with optical power meters to a precision of better then $2 \%$.

The light outputs recorded for four laser channels of a transmitter are shown in Fig. 4.a. In this test the irradiation was performed in five-minute intervals with one minute beam-on at a dose rate of $150 \mathrm{krad} / \mathrm{min}$ for laser diodes (GaAs), followed by a 4 minute beam-off period. The transmitter was chilled by a thermo-electric cooler with the substrate temperature maintained at $24.0^{\circ} \mathrm{C}$. During the one-minute irradiation, the proton beam was intense and the transmitter was heated up to $24.8^{\circ} \mathrm{C}$ at the substrate. The temperature was quickly restored in two minutes after the beam was shut off. The difference caused a few percent deviation in light outputs, seen in each irradiation cycle. The light outputs were recorded for twelve irradiation cycles, for a total dose of $1.8 \mathrm{Mrad}$. The measurement was extended for 24 hours to monitor annealing effect. The recovery in light output is about ten percent in a day.

The light degradation is approximately linear to the dose. It is consistent with the dominant bulk damage estimation. A linear fit was performed for each laser diode channel and the fit values at 200 and $400 \mathrm{krad}$, normalized to the original light output, are plotted in Fig. 4.b and c, respectively, for channels of three irradiated modules. The ratio of light loss for channels of a transmitter is relatively uniform. At $200 \mathrm{krad}$, the light degradation is about $10 \%$.

The radiation tolerance was also studied with $63.3 \mathrm{MeV}$ protons at the Crocker Nuclear Laboratory at UC Davis [8]. The fluence rate was $4.2 \times 10^{9}$ protons $/ \mathrm{cm}^{2} \mathrm{~s}$ $(0.56 \mathrm{krad} / \mathrm{s})$ over a uniform beam spot $7 \mathrm{~cm}$ in diameter, fallen by only $2 \%$ at a radius of $2 \mathrm{~cm}$. The beam fluence was monitored with a secondary emission device calibrated by a Faraday cup with an accuracy of better than $5 \%$. The transmitters were irradiated as integrated components on the silicon front-end Port Cards. Two Port Cards were irradiated for a total dose of 200 and 400
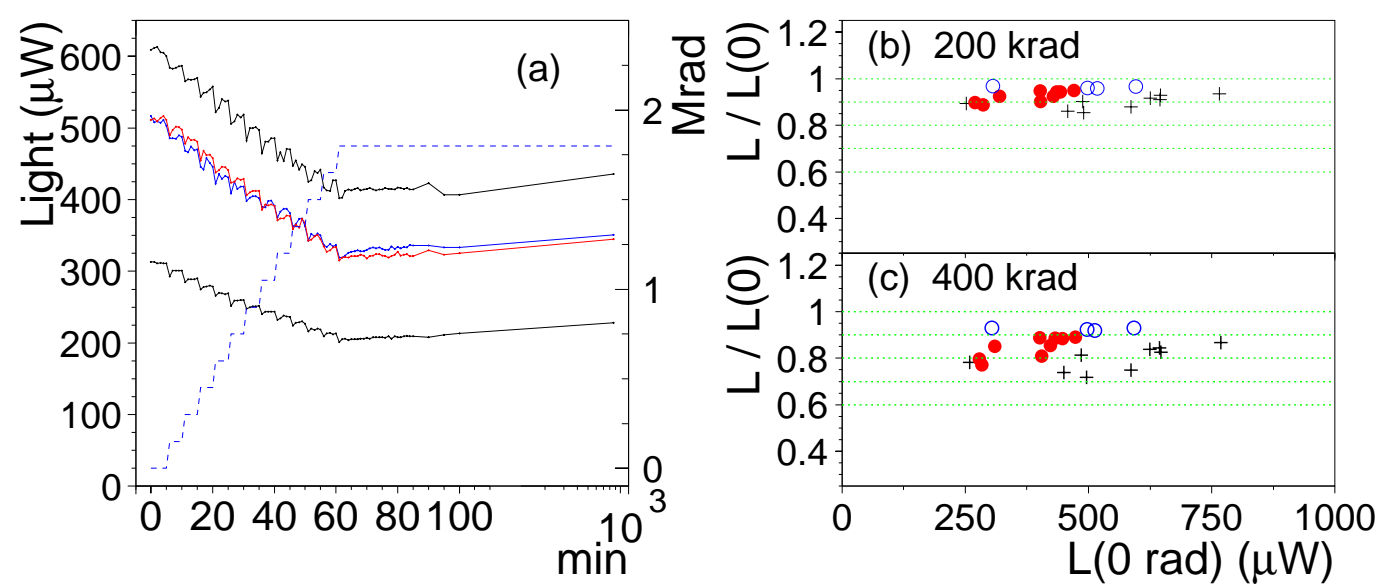

Fig. 4. (a) Light output measurements in time for an irradiation test at INER. The cumulative dose is shown by the dashed line labeled on the right. Ratio of light outputs at (b) $200 \mathrm{krad}$ and (c) $400 \mathrm{krad}$ versus the measurements prior to the irradiation. Channels of a transmitter are distinguished by the same type of mark. 
krad, respectively. The average laser light outputs measured after the irradiation reduced to $87 \pm 8 \%$ and $80 \pm 20 \%$, respectively.

At CDF the transmitters are mounted on the Port Cards on the outer ring of the silicon tracker. A Port Card has five transmitters reading five silicon ladders configured in a wedge. The transmitter pigtails were routed out of the CDF central tracker volume and were connected to $22 \mathrm{~m}$ long fiber ribbon cables to receivers on the Transition Module for the Fiber Optic Interface Board [9]. In total 570 DOIM module pairs were implemented for the CDF silicon tracker readout. After installation, many transmission errors had occurred and were treated for the main causes due to light saturation and bad electronic contacts. The light outputs for channels of a laser-diode array is uniform, however, the large deviation in light coupling to the pigtail fibers causes a few hundred $\mu \mathrm{W}$ in difference between channels of a module. This feature has largely reduced the dynamic range for setting the operation voltage and temperature.

The DOIM performance is constantly monitored. In the first year operation, the occurrence of bit-error has been maintained down to a few percent in modules. For long term reliability, the validation studies suggest that the radiation tolerance can meet the DOIM design specification.

\section{References}

[1] CDF Collab., "The CDF-II Detector Technical Design Report", FERMILABPub-96/390-E;

S. Nahn, "Status of CDF Silicon", Vertex 2002, these proceedings.

[2] M. Chu et. al., "Dense Optical Interface Module (DOIM)", Fermilab Electronic Systems Engineering document TWN-SVX-960628, CDF Note 3865, March 1996.

[3] Telecommunication Laboratories, Chunghwa Telecom Co. Ltd., Yangmei, Taoyuan 326, Taiwan.

[4] austriamicrosystems AG, A-8141 Schloss Premstätten, Austria.

[5] Plasma Optical Fibre, B.V., Eindhoven, The Netherlands.

[6] D. Husby, Fermilab Electronic Systems Engineering document ESE-SVX-950222, 21 October, 1997; http://www-ese.fnal.gov/projects/svx/bert/Xbert.htm.

[7] R.J. Tesarek et al., "Radiation field in the CDF Tracking Volume", CDF note 6060, RESMDD02 conference proceedings, to be published in Nucl. Instr. and Meth. A.

[8] J. Andresen et al., "Radiation Hardness of the Compact Port Card for the CDF Silicon Tracking Detector Upgrade", CDF note 5535.

[9] V. Pavlicek, "Transition Module for the Fiber Interface Board", Fermilab Electronic Systems Engineering document ESE-SVX-960624, 4 March, 1998;

K. Woodbury, "Fiber Interface Board", ESE-SVX-951010, 11 February, 1997. 